Voix et Images

volxetimages

\title{
Essai de typologie du discours pamphlétaire québécois
}

\section{Bernard Andrès}

Volume 1, numéro 3, avril 1976

Gérard Bessette

URI : https://id.erudit.org/iderudit/200041ar

DOI : https://doi.org/10.7202/200041ar

Aller au sommaire du numéro

Éditeur(s)

Les Presses de l'Université du Québec

ISSN

0318-9201 (imprimé)

1705-933X (numérique)

Découvrir la revue

Citer cet article

Andrès, B. (1976). Essai de typologie du discours pamphlétaire québécois. Voix et Images, 1(3), 417-431. https://doi.org/10.7202/200041ar d'utilisation que vous pouvez consulter en ligne.

https://apropos.erudit.org/fr/usagers/politique-dutilisation/ 


\section{Essai de typologie du discours pamphlétaire québécois}

Nous voulons remplacer avec toute la population cette société d'esclaves par une société libre fonctionnant d'elle-même et pour elle-même, une société ouverte sur le monde.

FLQ, octobre 1970

II suffit de remplacer le roi par vous, bon peuple, pour que vous-mêmes vous soyez suffisants; il faut ouvrir la France au monde entier et prêcher la Révolution sur toutes les frontières.

Robespierre, 1790

Brochure, tract, prospectus, libelle..., définissent communément le réseau analogique du pamphlet. Notre première démarche étant d'établir le corpus le plus vaste possible, nous tenterons d'élargir cette conception du pamphlet à tout type d'écriture outrée décriant pour la changer une situation révoltante pour l'auteur'.

Cette ouverture du concept repose sur le «décrier»qui dépasse le niveau purement dénotatif (décrire, ça peut n'être que constater, accepter ; décrier peut aboutir a l'énoncé performatif : "j'accuse").

Retenons pour l'instant dans le pamphlet, quelque chose de bref et de vigoureux, marqué par l'outrance d'un discours dont on se méfie (si l'on en est l'objet), ou auquel on n'accorde habituellement que peu d'intérêt à cause de son manque de "sérieux" ou de son caractère subversif :

Sorti de là, je me trouvai sur le grand degré avec $M$. Arthus Bertrand, libraire, un de mes jurés, qui s'en allait díner, m'ayant déclaré coupable. Je le saluai; il m'accueillit, car c'est le meilleur homme du monde, et chemin faisant, je le priai de me vouloir dire ce qui lui semblait à reprendre dans le Simple Discours condamné. Je ne l'ai point lu, me dit-il; mais c'est un pamphlet, cela me suffit. Alors je lui demandai ce que c'était qu'un pamphlet, et le sens de ce mot qui, sans m'être nouveau, avait besoin pour moi de quelque explication. C'est, répondit-il, un écrit de peu de pages comme le vôtre d'une feuille ou 
deux seulement. De trois feuilles, repris-je, serait-ce encore un pamphlet? Peut-être, me dit-il, dans l'acception commune; mais proprement parlant, le pamphlet n'a qu'une feuille seule; deux ou plus font une brochure. Et, dix feuilles? quinze feuilles? vingt feuilles? Font un volume, dit-il, un ouvrage. [...]

Si, au lieu de ce pamphlet sur la souscription de Chambord, j'eusse fait un volume, un ouvrage, l'auriez-vous condamné? - Selon. J'entends, vous l'eussiez lu d'abord pour voir s'il était condamnable. - Oui, je l'aurais examiné. - Mais le pamphlet, vous ne le lisez pas! - Non, parce que le pamphlet ne saurait être bon. Qui dit pamphlet, dit un écrit tout plein de poison. - De poison ? - Oui, monsieur, et du plus détestable, sans quoi on ne le lirait pas. - S'il n'y avait du poison? - Non, le monde est ainsi fait; on aime le poison dans tout ce qui s'imprime. Votre pamphlet que nous venons de condamner, par exemple, je ne le connais point, je ne sais, en vérité, ni ne veux savoir ce que c'est, mais on le lit; il y a du poison. M. le procureur du roi nous l'a dit, et je n'en doutais pas. C'est le poison, voyez-vous, que poursuit la justice dans ces sortes d'écrits. Car autrement la presse est libre; imprimez, publiez tout ce que vous voudrez, mais non pas du poison. Vous avez beau dire, messiours, on ne vous laissera pas distribuer le poison. Cela ne se peut en bonne police, et le Gouvernement est là qui vous en empêchera bien ${ }^{2}$.

Au Québec, cette indifférence n'est peut-être pas étrangère à l'anglicisme "pamphlet», désignant la brochure publicitaire ${ }^{3}$. Elle s'explique quand même assez mal quand on songe à la tradition polémiste des déclarations électorales au Pays, et, plus généralement, à la place du discours politique, de l'éditorial et des mandements épiscopaux depuis la Conquête:

Un pays conquis, disait L. Rièse, éveille l'éloquence, les diatribes,

le sens de l'humour ou de l'ironie, les tirades satiriques, la force vengeresse, une opinion enfin ${ }^{4}$.

Sans remonter au Déluge, les Relations des jésuites ne présententelles pas un caractère exagéré, magnifié (publicitaire?), dans leurs adresses aux supérieurs, bienfaiteurs, et missionnaires à venir de vieille France (Roger Duhamel parle même de "propagande")?

Nous tenterons ici de jeter des bases pour l'étude du pamphlet québécois, tant au niveau du corpus que de la méthode d'analyse.

\section{LE CORPUS}

\section{Pour une bibliographie critique}

Le corpus de nos recherches s'est limité jusqu'à présent aux seules années 1945-1971. Les notes qui suivent s'appuient modestement sur un certain nombre de ces écrits. Il s'agissait au départ d'un essai de comparatisme entre les écrits de cette période, et ceux de la Révolution française. Une Bibliographie que nous avons constituée avec une vingtaine d'étudiant(e)s, regroupe près de cent quatre-vingts textes. Elle reste bien insuffisante, et seul l'établissement d'une vaste et rigoureuse bibliographie critique permettrait de progresser dans ce domaine. 
On pourrait partir des premières interventions de parlementaires québécois contemporains de la Révolution française, pour rejoindre les discours de Papineau, Mercier, Bourassa (l'autre) et L. Groulx, par exemple. Le journalisme présenterait évidemment une excellente source, quand on sait le caractère frondeur qui l'a marqué dès les débuts ( $\mathrm{Mgr}^{\mathrm{r}}$ Hubert s'en plaignait déjà, lui reprochant sa « licence"!).

Il faudrait considérer les articles de la Gazette de Montréal (1778), qui valurent la prison à Jautard et Mesplet (voir de ce dernier: I'Adresse du Congrès aux habitants de la Province de Québec).

Également: le Courrier de Québec (1788); certains textes du Canadien (1806-1893); la Gazette de Trois-Rivières (1817-1822), et surtout la Minerve (1826-1899), avec les interventions d'Étienne Parent; le Fantasque (1837), de Napoléon Aubin; I'Avenir (1847); le Pays (1852); le Devoir (des années 1910); l'Ordre d'Asselin et le Jour de Harvey ${ }^{5} .$.

Les revues, de même, constitueraient une mine: Canada-Revue (1891-1892); Vivre (1934); la Relève et la Nouvelle Relève, à partir de 1941, Relations (du temps d'Asbestos), Cité libre, Révolution québécoise, 'Parti pris, Québec-Presse, Mainmise, Presqu'Amérique, Hobo-Québec, Liberté, etc. Enfin, une attention toute particulière reviendrait à des textes-jalons plus élaborés; manifestes d'écoles littéraires (textes de l'Institut canadien, de l'Hexagone), artistiques (le Refus global), écrits politiques (Manifeste du F(Q), ou cuvres individuelles marquantes: l'Avenir de notre bourgeoisie (1939), d'Albert Pelletier; la France et nous, de Robert Charbonneau; le Journal d'un Inquisiteur, de Gilles Leclerc; les Insolences du Frère Untel; le Joual de Troie, Nègres blancs d'Amérique, etc. ${ }^{6}$.

On voit que cette bibliographie exigerait de rigoureuses recherches. Comment la rendre "critique"?

N'oublions pas le stade auquel on se situe encore: définition du. plus large corpus, en vue d'une analyse subséquente. La démarche est inductive. II s'agit de trouver des textes supposément pamphlétaires, c'est-àdire, admis comme tels à leur parution ou par la suite. On retient momentanément pour pertinent le consensus socio-culturel d'une synchronie donnée (dates de parution d'un texte) ou d'une diachronie limitée (postérité plus ou moins immédiate d'une œuvre).

Ces textes dépistés, une première lecture permet de les classer grossièrement en fonction de leur source et de leur idéologie.

\section{LA SOURCE}

Dans un travail analogue, nous avons établi une grille assez commode qui rend compte de la plupart des textes à partir de neuf combinaisons fonctionnant sur quatre éléments :

- l'identification de l'auteur (ou du groupe)

- la popularité de l'auteur 
- la représentativité de l'auteur

- la popularité de ce groupe représenté.

Un cinquième élément concerne la diffusion. Plus difficilement quantifiable, elle donne cependant d'intéressantes indications.

On obtient ainsi un encodage à neuf combinaisons, illustré par les tableaux I et II. Quelques remarques s'imposent sur la nécessité du recours à ces éléments. Nous nous en tiendrons ici aux deux premiers: l'identification et la popularité.

\section{Identification de l'auteur}

Négligeant à ce niveau de l'analyse la distinction auteur-locuteur, nous définirons l'identification comme la coïncidence entre la dénomination de l'auteur et celle du signataire.

Le cas d'un auteur non identifié pose le problème du pseudonyme littéraire ou du "nom de combat" (les Insolences du Frère Untel, le Carnet d'un croyant perplexe, le Journal d'un Inquisiteur, etc.). On peut distinguer ici deux types de censure: “externe" ou “interne", selon qu'elle dépend d'une pression étrangère, ou que l'auteur se l'impose par intérêt personnel.

Dans la censure "externe" (euphémiquement qualifiée d' «auto-censure»), le pamphlétaire prend ses distances (et ses précautions) "de son propre chef "... de crainte de représailles. On peut aussi lui imposer de se démarquer par rapport à une collectivité qui s'en désolidarise. Il doit alors parler "en son nom propre" (même d'emprunt), gommant au niveau de la signature toute appartenance au dit groupe. Citons le cas particulier du Frère Untel qui, mimant l'anonymat, affiche habilement sa filiation cléricale... et l'insolence de ses propos.

La censure "interne" est celle du sujet qui, établissant une hiérarchie dans ses productions, ménage les plus "nobles" d'entre elles en gardant l'anonymat sur les autres: erreurs de parcours, fautes de jeunesse... ou littérature alimentaire (cf. les 100,000 verges, d' "Apollinaire" et J'irai cracher sur vos tombes, de "Boris Vian Sullivan").

Les stratégies imposées par la censure sont parfois si subtiles qu'on a du mal à en définir certaines. Que dire des écrits de Mai 1968 attribués par solidarité à Cohn-Bendit (alors expulsé en Allemagne) ? Solidarité cristallisée dans le slogan: "Nous sommes tous des Juifs allemands". Au Canada, lors de mouvements contestataires indiens, des tracts rédigés par des blancs étaient signés de pseudonymes cris. Comble de la "faussereprésentation"?

S'identifier, à la clôture du pamphlet, c'est apparemment assumer son discours. En fait, ce n'est qu'une des façons de le faire, pas la meilleure, ni la plus efficace. Nous verrons que la notion de risque doit être pondérée par celle de la popularité éventuelle du sujet et/ou du groupe auquel l'auteur est affilié. 
TABLEAU I

A- Identification

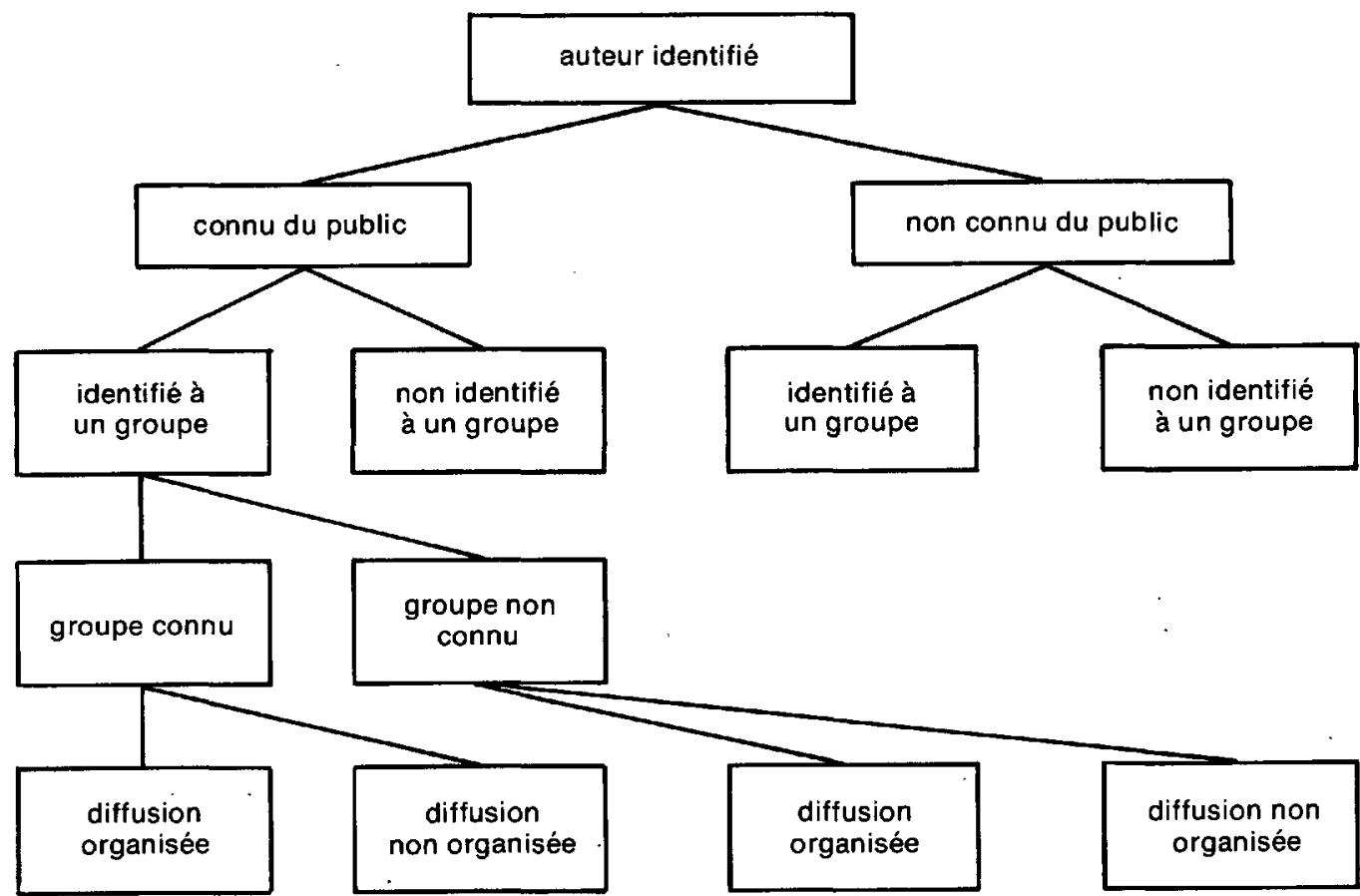


TABLEAU ॥

\begin{tabular}{|c|c|c|c|c|c|c|c|c|}
\hline CODES & $\begin{array}{l}\text { auteur } \\
\text { identifié }\end{array}$ & $\begin{array}{c}\text { auteur non } \\
\text { identifié }\end{array}$ & $\begin{array}{l}\text { connu du } \\
\text { public }\end{array}$ & $\begin{array}{l}\text { non connu } \\
\text { du public }\end{array}$ & $\begin{array}{l}\text { identifié } \\
\text { à un groupe }\end{array}$ & $\begin{array}{l}\text { non identifié } \\
\text { à un groupe }\end{array}$ & $\begin{array}{l}\text { groupe } \\
\text { connu }\end{array}$ & $\begin{array}{l}\text { groupe } \\
\text { non connu }\end{array}$ \\
\hline Source I & $x$ & & $x$ & & $x$ & & $x$ & \\
\hline Source II & $x$ & & $x$ & & $x$ & & & $x$ \\
\hline Source III & $x$ & & $x$ & & & $x$ & & \\
\hline Source IV & $x$ & & & $x$ & $x$ & & $x$ & \\
\hline Source V & $x$ & & & $x$ & $x$ & & & $x$ \\
\hline Source VI & $x$ & & & $x$ & & $x$ & & \\
\hline Source VII & & $x$ & & $x$ & $x$ & & $x$ & \\
\hline Source VIII & & $x$ & & $x$ & $x$ & & & $x$ \\
\hline Source IX & & $x$ & & $x$ & & $x$ & & \\
\hline
\end{tabular}


Notons pour l'instant qu'à rebours d'un cas précédemment cité, le sujet peut cautionner son texte pamphlétaire en exploitant la renommée acquise par d'autres de ses écrits moins sujets à... caution. La notoriété déjoue la censure, mais l'efficacité en pâtit, minée par une récupération rétrospective.

Dans le même ordre d'idées, le cas du sujet lancé par le coup d'éclat d'un texte tapageur (il n'y a pas de mauvaise publicité)... et récupéré plus tard par quelque officine gouvernementale.

Retenons que l'efficacité de cette écriture n'est pas intimement liée à l'identité de son auteur, et que bien souvent, hélas, le danger du pamphlet, c'est le pamphlétaire. Tel texte séduisant par sa vigueur et sa férocité, se trahit par les mobiles du locuteur. Mais on touche là au problème de la popularité acquise ou cherchée de celui par qui le scandale arrive.

\section{La popularité de l'auteur}

Il est difficile de porter un jugement précis sur les mobiles de l'auteur ou du groupe qu'il représente. On se limitera pour apprécier le degré de popularité, à un critère plus quantitatif que qualitatif, basé sur la fréquence des interventions du sujet sur la scène nationale ou provinciale, à travers toutes sortes de media.

L'étude de cet élément présente un intérêt certain dans la mesure où il permet de distinguer deux grands types d'écrits pamphlétaires:

1. Les textes à fonction phatique élevée où le locuteur, inconnu, cherche surtout à établir et à maintenir une communication valable avec l'allocutaire:

Je $m$ 'adresse à des hommes libres, mais it peut arriver que ce soit là une utopie parmi bien d'autres [...] $\rightarrow$ les hommes libres feront écho à ma voix $[. ..] \rightarrow$ Si les hommes libres ne sont aptes qu'à plagier les réactions des esclaves, de quel droit se disent-ils libres [...] $\rightarrow$ Mes lecteurs essaieront donc de comprendre avant de s'indigner $[. . .]^{7}$.

Cette tension vers le lecteur, l'outrance de la fonction phatique qui en résulte, nuisent considérablement à la portée des attaques.

2. On remarque d'autre part des textes à fonction conative élevée, où l'auteur se contente d'agir sur un public qui le reconnaît, dont il ne doute pas de l'attention. Cette assurance peut provenir de conditions d'énonciation privilégiées: la communication orale avec l'interlocuteur tout proche; le discours institutionalisé des parlementaires (où les autres - quand ils sont là - sont censés écouter); ou encore les éditoriaux adressés aux abonnés de revues, de journaux (Marat s'adressait seul aux Parisiens dans l'Ami du peuple).

L'équilibre, il est vrai, reste précaire. Le 9 Thermidor de l'an II (27 juillet 1794), Saint-Just s'adresse à la Convention pour la dernière fois: les conjurés lui coupent la parole (pour commencer...). Pour les grandes figures de la Terreur, toute baisse de popularité est fatale. Elle annonce la chute du Chef. 
Pour en finir nous aussi avec nos auteurs (identifiés ou non), disons que la classification par la source permet, dès la première lecture, d'encoder le texte à l'aide d'une formule simple portée sur la fiche de lecture:

exemple: "S. Ill" désigne la troisième combinaison des sources, c'est-à-dire: auteur identifié, connu du public, non identifié à un groupe.

\section{L'IDÉOLOGIE}

Un dernier type d'éléments pourrait apparaître sur la fiche d'information de chaque texte, pour parfaire l'aspect critique de la bibliographie. II s'agirait de mentionner les codes connotatifs ${ }^{8}$ véhiculés par le texte et qui confèrent à celui-ci la coloration idéologique évoquée plus haut.

II ne s'agit pas de résumer le plan dénotatif du texte, mais d'en indiquer l'orientation générale.

La sollicitation directe ou indirecte marque la plupart des clôtures pamphlétaires: appel à l'action, au soutien moral ou matériel (voire pécuniaire). On reviendra plus loin sur cette dynamique des forces illocutoire (donner un ordre) et perlocutoire (exécuter l'ordre) de l'énonciation pamphlétaire. 'Il s'agit donc de signaler brièvement les moyens mis en cuvre au niveau connotatif pour parvenir à cette sollicitation.

On rencontre le plus fréquemment dans l'écriture pamphlétaire:

le code scientifique (sc.) qui dote le texte d'un vernis de technicité propre à épater le lecteur non spécialiste (les discours électoraux contemporains puisent dans le lexique technocratique la poudre aux yeux des chiffres et pourcentages destinés à leurrer - pour parler poliment - le citoyen);

le code rhétorique (rh.) qui prend appel sur toutes les règles traditionnelles du dire (tropes, figures, etc.), ne fait souvent qu'afficher une éloquence dont il abuse... pour abuser (amuser?) le lecteur:

II nous suffit d'ouvrir à quelques milliers d'âmes les rares fenêtres qui donnent sur l'horizon clair du monde. Les autres, incarcérées dans le noir, sous les souffles humides et délétères de l'ignorance, finiront, elles aussi, par monter vers la clarté ${ }^{9}$.

le code de la communication qui renvoie aux appels, questions, ordres, conseils au lecteur. C'est l'acte “illocutoire»d'Austin, par lequel la phrase, performative, se pose elle-même comme acte, en vue d'une prise de conscience ou d'une action à venir, conséquence seconde ( perlocutoire") de sa parole:

Travailleurs du Québec, commencez dès aujourd'hui à reprendre ce qui vous appartient; prenez vous-même ce qui est à vous. Vous seuls connaissez vos usines, vos machines, vos hôtels, vos Universités, vos syndicats; $n$ 'attendez pas d'organisation miracle ${ }^{10}$.

les codes socio-culturel, politique et historique qui sont de loin les plus fréquents dans nos écrits qui énoncent des "vérités" sans toujours les prouver, par simple allusion à des traits de civilisation, des mœurs politiques ou des référents historiques considérés comme évidents, incontestés et incontestables. 
Débusquer ces codes connotatifs, c'est lire entre les lignes une idéologie souvent différente de celle affichée au niveau dénotatif. Relire à ce propos les conseils prodigués par les banques à leurs braves clients, dans «leur» propre intérêt. Ré-écouter aussi les brillants discours électoraux des veilles-qui-chantent. Scruter dans cette optique les discours adressés au peuple à travers le "Cours de personnalité et de philosophie populaire", dispensé pour l'Institut Le Royer par son directeur, en 1957:

Je refuse de laisser la notion de renouvellement à la gauche, pour la bonne et simple raison que si vous posez le renouvellement à gauche, la notion de renouvellement, elle, n'est pas celle de n'importe quel mouvement! Pour renouveler et pour rajeunir, il faut se mouvoir au sein de la tradition, progresser selon la nature des choses, et aimer la liberté, selon le bien intelligible donné par la vérité.

A ce moment-là, messieurs, si vous aimez l'épanouissement de la liberté orientée par la vérité, vous n'êtes pas à gauche, vous êtes autant à droite qu'à gauche; vous êtes même de façon prépondérente à droite, puisque vous admettez l'orientation de la vérité ${ }^{11}$.

Pour s'y retrouver dans cet imbroglio lexicologique très révélateur où chacun engrosse la langue au gré de ses fantaisies oratoires, on s'en tiendra à une terminologie plus simple; la distinction traditionnelle droite - gauche:

Le combat politique oppose ceux qui sont à peu près satisfaits de l'ordre existant, qui veulent le conserver; et ceux à qui cet ordre ne convient pas, qui veulent le changer ${ }^{12}$.

Par «ordre», nous entendons celui du système socio-économique du capitalisme occidental, et plus particulièrement, nord-américain. Nous introduirons les variations idéologiques suivantes:

D $\begin{cases}\text { Extrême-Droite (+D): } & \begin{array}{l}\text { maintenir l'ordre à tout prix (en s'oppo- } \\ \text { sant à toute atteinte, même mineure, au } \\ \text { système). } \\ \text { ordonner "dans l'ordre" justices et injus- } \\ \text { tices (céder sur des détails en mainte- }\end{array} \\ \text { G. } \begin{cases}\text { nant l'essentiel). } \\ \text { changer l'ordre “dans l'ordre" (réformes } \\ \text { ponctuelles respectant le système électo- } \\ \text { Extrême-Gauche (+G): }\end{cases} \\ \text { ral). } \\ \text { renverser l'ordre rapidement et par la for- } \\ \text { ce (sans tenir compte du jeu électoral). }\end{cases}$

On voit aisément dans ces variantes où se situera l'outrance pamphlétaire qui s'accommode mal des modalisations de l'écriture centriste. Deux étudiants avaient bien cerné ce problème en remarquant, dans un style luimême assez pamphlétaire:

Les textes ainsi produits seront truffés de nuances temporisatrices, de bon-ententisme, d'ironie empreinte de collégialité. Dans cette situation inconfortable de l'entre-deux chaises, nous trouvons tous les Ryanistes de tous poils exposant leur texte au compteur Geiger de l'autocensure, s'astreignant au dogme de la sacro-sainte objectivité, se ver- 
mifugeant au code d'éthique de l'élite et de la tempérance petite-bourgeoise; on ne peut s'attendre qu'à des textes délavés, dilués, distillés, désamorcés, émasculés, dénués de toute la passion fiévreuse et du généreux spontanéisme avec lequel se manifestaient par exemple les pamphlétaires célèbres ${ }^{13}$.

Pour ma part, j'accepterais volontiers dans le corpus des textes de droite qui, anodins en apparence, miment l'innocence qu'ils ne font que dénoter... tout en connotant une outrance idéologique surprenante. Jacques Guillette et Serge Phaneuf remarquent que "ce qui vole la vedette au F.L.Q., c'est le «Texte des Mesures de Guerre" [...], plus subversif que le Manifeste du F.L.Q. Iu le 11 octobre 1970 à la télévision ${ }^{14}$ ". Ne retenir que l'outrance dénotée (attaques nominales, invectives, exaltation du locuteur...), c'est trop limiter le champ du pamphlet. Les plus efficaces en dernière analyse, sont peut-être les textes produits par l' "establishment ", qui recèlent une plus forte charge explosive.

Ils se présentent effectivement voilés et désarment la prudence du lecteur, là où les pamphlets de gauche se désignent comme des textes outrés, affichent leur code. Ces derniers, prévenant le lecteur, désamorcent leur propre charge... ou ne se font lire que par des sympathisants. Borduas écrit à propos de sa découverte du pamphlet en France:

Des œuvres révolutionnaires, quand par hasard elles tombent sous la main, paraissent les fruits amers d'un groupe d'excentriques. L'activité académique a un autre prestige à notre manque de jugement ${ }^{15}$.

II est vrai que l'efficacité, le rendement, ne sont pas des critères majeurs pour le pamphlétaire qui les dénonce précisément dans le système à abattre. Toujours est-il que si l'impact du pamphlet s'apprécie sur le lecteur non sympathisant, le meilleur pamphlet n'est pas un pamphlet!

D'où la contradiction interne de ce genre littéraire. Seule issue logique (et politique): imposer par la force l'écrit. On retrouve alors la «clôture du sang " dont parle Barthes au sujet de la Terreur en France:

Si la Révolution n'a pas modifié les normes de [l'écriture classique], parce que le personnel pensant restait somme toute le même [...], les conditions exceptionnelles de la lutte ont pourtant produit au sein même de la grande Forme classique, une écriture proprement révolutionnaire, non par sa structure, plus académique que jamais, mais par sa clôture et son double, l'exercice du langage étant alors lié, comme jamais encore dans l'Histoire, au Sang répand ${ }^{16}$.

Le premier communiqué de la cellule Libération du FLQ exige la diffusion de son Manifeste, et offre comme seule distraction au diplomate sequestré... la lecture de Nègres blancs d'Amérique!

Le locuteur, son identification, l'idéologie véhiculée; voilà l'esprit dans lequel pourrait être constitué le corpus.

Cette tâche accomplie, comment débrouiller l'écheveau des textes rassemblés? Une méthodologie s'impose. 


\section{TYPOLOGIE DU PAMPHLET}

\section{Analyse stylistique de l'énoncé et de l'énonciation}

Connaître un type d'écriture, c'est recenser un certain nombre de textes présentant des éléments analogues, les comparer, en tirer les combinaisons dominantes qui définiront par leur récurrence le type cherché. C'est dire qu'en ce qui concerne le pamphlet, discours peu étudié ou trop aisément assimilé à l'essai, l'attention se portera sur des agencements discursifs relevant d'une stylistique de l'énoncé et de l'énonciation.

On ne se défendra pas d'aborder dans l'étude du référent les conditions socio-historiques d'émergence textuelle : elles permettront même 'd'apprécier la portée de telle ou telle combinaison et de pondérer nos appréciations. Le Refus global de Borduas n'a pas l'éclat des outrances sadiennes sous la Révolution, mais il appelle à un «règlement final des comptes 17 " peut-être plus dangereux pour la sécurité de l'auteur à l'époque où il le prononce, que l'exhortation de Sade: Français, encore un effort... Se repose tout le. problème de la censure. Crainte, bravée ou déjouée, elle est latente dans chaque pamphlet. Elle en détermine en creux la structure, formant ou déformant l'écrit suivant l'attitude adoptée par le locuteur à son égard.

\section{L'ÉNONCÉ DU DISCOURS PAMPHLÉTAIRE}

II s'agit des aspects proprement verbal, syntaxique et sémantique du texte.

L'étude du niveau verbal porte sur le lexique choisi, les caractéristiques rythmiques et mélodiques et la disposition typographique du texte.

L'essai, auquel on rattache souvent le pamphlet, se présente comme le genre le plus libre à ce point de vue. L'auteur y a toute latitude dans le choix du lexique en fonction de l'effet à obtenir, ou éventuellement, de la démonstration à effectuer. En fait, on démontre moins dans l'essai (comme on le ferait dans la thèse), qu'on expose en amateur des opinions qui n'engagent que soi, sur un sujet qu'on ne songe pas à épuiser:

Ces pages ont été préparées pour la radio. De là leur état apparemment fragmentaire. Elles se présentent comme des contemplations successives dont la trame n'est peut-être pas suffisamment soulignée,

avertit Ernest Gagnon en tête de l'Homme d'ici ${ }^{18}$.

Le pamphlet, plus court, nécessite un choix de termes plus marquants, un lexique plus vif qui fixera le temps de la lecture, l'attention de l'allocutaire (fonction phatique). II s'agit de dénoncer et de convaincre vite. La disposition typographique, l'illustration et le recours à l' "icône" (au sens peircien) y sont pour beaucoup. L'aspect matériel, tactile, du paimphlet frappe à un tel point l'attention que Pauvert lance la collection "Libertés" en petit format de poche (aisément dissimulable "sous le manteau" du contestataire en mal de censure), et sur papier brun de type «emballage»! 
Au niveau syntaxique, le pamphlet se distingue de l'essai par la stylisation des relations logiques entre propositions. Là où l'essayiste peut développer un système démonstratif, le pamphlétaire ne retient (et n'affiche) que des marques, des symboles verbaux de causalité, conjonction, disjonction, inclusion, etc. On connote.le raisonnement plus qu'on ne l'effectue.

Toujours au niveau syntaxique, une recherche serait à faire sur les relations spatiales entre propositions (symétrie, opposition, gradation), qui rappellent étrangement par leur fréquence et la recherche de l'effet à obtenir, des relations analogues, propres à l'écriture poétique.

Au plan sémantique, si l'on considère d'abord la question de la représentation, on s'aperçoit que notre discours balance systématiquement entre la dénotation la plus poussée et les plus vagues connotations. Le locuteur reproche directement des faits précis en nommant l'ennemi, mais son discours ne s'en tient pas là. C'est tout à la fois l'acte d'accusation et la condamnation sans appel, au nom d'idéaux, de vérités implicites connotées par des mots clés du genre:

- “Américain ", ou «impérialiste ", dans un texte d'extrême-gauche;

— "Canadien/an" ou "fédéraliste " dans un texte séparatiste;

— "Moscou » ou «libéral " dans un texte de Rumilly.

Ce type d'écriture politique, explique Barthes, «fait l'économie d'un procès: il n'y a plus aucun sursis entre la dénomination et le jugement ${ }^{19}$ ". T. Todorov définit la "figuralité " comme «la disponibilité du langage à se laisser percevoir en lui-même. Toutes les figures de discours apparaissent dans l'entreprise de démolition qu'est le pamphlet. L'outrance verbale fait feu de tout trope, sans aucune déférence aux vieilles règles d'usage de l'éloquence. Techniques de persuasion et d'amplification se combinent selon de nouvelles lois (sauf chez quelques rhéteurs qui ordonnent quelque peu leur discours).

L'emphase est reine... et pourtant on ne sourit pas quand la situation pêche elle-même par ses excès : elle exige ce langage outré, comme par mimétisme:

Tuons Saint Jean-Baptiste! Brûlons le carton-pâte des traditions avec lequel on a voulu mythifier notre esclavage. Apprenons l'orgueil d'être hommes. Affirmons fortement notre indépendance. Et écrasons de notre liberté robuste le paternalisme compatissant ou méprisant des politiciens, des papas-patrons et des prédicateurs de défaites et de soumissions... ${ }^{20}$.

Une dernière caractéristique de ce type d'énoncé : sa propension à l'intertextualité, son ouverture sur d'autres énoncés qui l'illustrent et le cautionnent. Toute référence littéraire, historique, politique ou d'actualité est bienvenue dans ce texte d'un locuteur isolé (comme individu ou comme groupe marginal), à l'affût de tout assentiment, même venu du passé. Les "phrases-sentences " de Gilles Leclerc, où Jean Marcel voit «les formes cristallisées de la colère", illustrent bien cette caractéristique, à travers le mélange des styles bas, moyen et élevé: 
Je comprends mal et je digère plus mal encore cette épouvante [...] des mercenaires de l'enseignement devant la pensée et la liberté intellectuelles, quand j'observe que l'une et l'autre ont assez rarement dans I'Histoire constitué la clientèle des tavernes, des bordels, des tripots, des hippodromes, des prisons et des émeutes. Penser c'est s'humaniser, et je ne sache pas que Shakespeare, Matisse, Papini ou Camus y aient moins réussi que Louis XIV, Al Capone, Churchill ou Laurier ${ }^{21}$ !

Les codes socio-historique, culturel, esthétique, etc., fonctionnent à plein, dans la plus vaste plurivalence sémantique.

\section{L'ÉNONCIATION DU DISCOURS PAMPHLÉTAIRE}

Nous terminerons cette approche stylistique par quelques remarques sur la façon dont le pamphlet se situe comme acte de parole, le locuteur prenant et assumant celle-ci, face à un interlocuteur à convaincre (après l'avoir trouvé et avoir su le garder).

L'énonciation, c'est un peu la mise en scène de la parole, et l'on comprend que la "théâtralité" de cette écriture se prête à cette grille d'analyse. Dès le Prologue au Journal d'un Inquisiteur, une forte actualisation se fait sentir :

Je m'adresse à des hommes libres [...] Je m'accroche pour le moment à cette présomption [...] Que celui qui est sans argent me lance la première pierre.

Mais l'allocutaire est encore loin, absent (Benveniste montre que le «il» est la "non-personne"). Le texte est de 1960. L'aube de la "conscientisation" du public québécois. Leclerc, aigri, «cherche un homme". Douze ans plus tard, Bergeron semble l'avoir trouvé. On passe du «il» au " tu». Dans Pourquoi une révolution au Québec (ce n'est pas une question !), le locuteur ouvre le texte à la Diderot, par une mise en situation dialoguée ou l'allocutaire est projeté à l'avant-scène:

- Quoi? Une révolution? Du sang dans les rues, des blessés, des morts [...] ? J'en veux pas.

- Crains pas. Elle viendra pas si la majorité des québécois la veulent pas $[\ldots]^{22}$.

Remontant à Gérin-Lajoie puis ré-utilisé par Duplessis contre Taschereau, le Catéchisme des électeurs fonctionne sur un mode analogue: l'énonciation est au premier plan. Un allocutaire pọse des questions innocentes (celles de l'électeur). Un locuteur y répond avec assurance et persuasion, définissant par ses propos une banque de réponses-types, sorte de manuel du parfait agent électoral ${ }^{23}$.

Signalons que dans tous ces textes, les styles émotif et évaluatif sont de mise. L'expression de la passion du locuteur provoque celle du lecteur:

Quand un homme parle de l'intelligence avec la passion que j'y mets, il y a le danger que les imbéciles l'accuse [sic] de pédantisme, qu'à cela ne tienne: je ne m'objecte pas à la vocation des ânes, ce serait gaspiller de la salive et du temps. Je poursuis donc ${ }^{24}$. 
C'est net, clair, précis, sans modalisations, ces bavures du discours centriste évoquées plus haut.

Je reviendrai pour finir aux définitions qui inauguraient cette étude. II s'agissait d'ouvrir au maximum la notion d'écriture pamphlétaire. C'est que l'énonciation de nombreux de ces textes ne manque pas de faire intervenir des bribes de discours narrativé. On dépasse le dialoguisme à la Diderot ou à la Bergeron. Le locuteur en vient à insérer les répliques dans une situation narrative avec personnages, décor et chronologie, un peu comme dans l'extrait du Pamphlet et des pamphlets reproduit plus haut: l'énonciateur' premier devient l'actant d'une séquence narrative = la rencontre-avec-le-jurésur-les-marches-du-Palais-de-justice.

Cependant (et c'est tout ce qui fait la différence entre le pamphlet et le roman), le passage narrativé n'est ici qu'un alibi du discours pour déjouer la censure. Par cette "déviation fictive", le ridicule est jeté sur l'interlocuteur Arthus Bertrand-un-de-mes-jurés. Plus loin dans le texte, un «je» assumera des propos encore plus subversifs. Gradation dans la prise en main de l'énonciation? II n'en est rien. Ce nouveau « je " réfère au «bon ami sir John Bickerstaff " (l'éditeur croit bon de préciser dans une note en bas de page: «Personne imaginaire»!). Le tour est joué: le locuteur initial ne faisait que traduire une lettre de John...

Qui parle à qui? Le système d'énonciation pamphlétaire est là pour empêcher d'y répondre.

Cette définition élargie n'a donc pas pour but de tout ramener à l'écriture pamphlétaire.

Le discours narratif présente à divers degrés les marques d'un locuteur brûlant de se prononcer sur le récit qu'il énonce. Ce n'est que la fonction idéologique plus ou moins latente dans toute cuvre romanesque. Une typologie du pamphlet tenant compte des éléments dominants évoqués dans cette étude, permettrait précisément de poser des limites entre les genres voisins. Et peut-être de répondre à cette question:

Par où commencer et jusqu'où irons-nous au Québec avec ce genre littéraire ainsi défini par Courier:

De l'acétate de morphine, un grain dans une cuve se perd, n'est pas senti, dans une tasse fait vomir, en une cuillerée tue, et voilà le pamphlet.

C'est grand comment, le Québec?

Bernard Andrès

Université du Québec à Montréal

1. ... ou décriant toute tentative de changement d'une situation choisie.

2. Paul-Louis Courier, le Pamphlet des pamphlets, 1824, Pauvert, 1965, p. 108 ss. 
3. Nous avons dans un premier temps considéré ce type d'écriture qui présentait des éléments analogues au pamphlet proprement dit. N.B.: le “NOUS" n'est pas de majesté; il désigne le groupe d'étudiant(e)s avec lequel j'ai pu amorcer cette étude et qui retrouvent ici, avec ma sympathie, des traces de nos recherches communes.

4. Cité par Laurent Mailhot dáns la Littérature québécoise, Paris, PUF, «Que saisje? $", 1974$, p. 15.

5. ...jusqu'à cette époque. II faudrait continuer jusqu'à nos jours...

6. Ces listes ne sauraient être exhaustives. Notons encore qu'elles devraient s'enrichir des écrits de "l'autre bord"; pêle-mêle: les Mandements de NN.SS. Bourget et Laflêche; le Rapport Durham; Pour la patrie (1895), de Tardivel; l'Ami du peuple, de l'ordre et des lois (1832-1840), de Leclère; The Quebec Mercury; Pages d'histoire politique, de Robert Rumilly; le texte des Mesures de guerre, de 1970; Place à l'homme, d'Henri Bélanger, etc.

7. G. Leclerc, Journal d'un Inquisiteur, Montréal, 1960; Jour, 1974. II s'agit de la page liminaire du Prologue. Les flèches marquent la réitération d'un appel désespéré à un lecteur digne du texte.

8. Pour les questions de connotation, voir Barthes, $S / Z$, Paris, Seuil, 1970, p. 13 à 16 , et "Analyse textuelle d'un conte d'E. Poe", dans Sémiotique narrative et textuelle. Paris, Larousse, 1973: « Le mot code lui-même ne doit pas être entendu ici au sens rigoureux, scientifique du terme. Les codes sont simplement des champs associatifs [...] l'instance du code, pour nous, est essentiellement culturelle: les codes sont des types de déjà-vu, de déjà-lu, de dejà-fait. " P. 50.

9. J.-C. Harvey, les Demi-Civilisés, 1934, Montréal, L'Actuelle, 1970, p. 77. Voir aussi les pages 89, 131-132, et 128-129 de ce texte qui s'est attiré les foudres d'un mandement épiscopal!

10. Manifeste du FLQ, lu à CKAC, le $7 / 10 / 70$.

11. André Dagenais (qui souligne), p. 19 de la Brochure Droite et Gauche. L'auteur associe dans son cours de Personnalité:

"a) 'lart de convaincre et de persuader dans les affaires;

b) la connaissance de la Personnalité et de ses ressources humaines". (Ici je souligne.)

12. Tout ceci reste encore schématique et se situe à un premier temps de la démarche: le corpus. Les catégories exposées n'ont qu'une valeur opératoire. La citation est de Maurice Duverger, Introduction a la politique, Paris, Gallimard, "Idées " $n^{\circ} 44$, p. 242.

13. Johane Belleau et Jacques Cordeau, session hiver 1975.

14. Travail de session, hiver 1975.

15. Refus global, Montréal, Parti pris, 1974.

16. Le Degré zéro de l'écriture, Paris, Seuil, 1953, p. 34.

17. Refus global, p. 17.

18. Montréal, HMH, 1963, p. 1. N.B.: Il s'agit pour l'instant de l'essai littéraire, non du scientifique.

19. Le Degré zéro de l'écriture, p. 38.

20. P. Vallières, Nègres blancs d'Amérique, Montréal, Parti pris, 1974, p. 24.

21. G. Leclerc, Journal d'un Inquisiteur, p. 190.

22. Éditions québécoises, 1972, p. 5.

23. Le Catéchisme des électeurs, 1851, puis 1935, S. Saint-Martin. Citons aussi le Manifeste du Crédit social, de L. Even.

24. Leclerc, op. cit., p. 98. La faute d'orthographe, à la troisième ligne, sera une faute d'imprimerie. II n'en est pas de même chez Bergeron: "NOTE SUR LES “FAUTES DE FRANCCAIS": "Toutes les fautes de Français de ce texte sont voulu [sic] et prémédité [re-sic]. II faut commencer quelque part à un moment donné à écrire comme du monde et cesser d'écrire comme l'exige la classe dominante pour perpétuer son règne." (Pourquoi une révolution au Québec, p. 177.) 